\title{
Neurological signs in relation to cancer in patients with asbestosis
}

\author{
Juhani Juntunen, Panu Oksa, Eero Pukkala, Pekka Laippala
}

P Oksa

Finnish Cancer

Registry, Liisankatu 21

B, FIN-00710 Helsinki, Finland

E Pukkala

Tampere School of Public Health, University of Tampere and Tampere

University Hospital, PO Box 607,

FIN-33101, Tampere,

Finland

P Laippala

Correspondence to:

Professor J Juntunen, LEL

Employment Pension Fund,

Palkkatilanportti 1,

FIN-00240 Helsinki,

Finland.

Accepted 27 May 1997

(Occup Environ Med 1997;54:746-749)

Keywords: cancer; nervous system; paraneoplastic; asbestosis; prospective study

Cancer has the unique property of simultaneous occurrence of seemingly independent disturbances in function of the nervous system (paraneoplastic nervous system involvement) ${ }^{1-3}$ The best known paraneoplastic neurological disease is peripheral neuropathy, which is usually a sensorimotor polyneuropathy sometimes resembling radiculopathy. ${ }^{4}$ Other disturbances of the nervous system have been reported to be associated with cancer, such as encephalopathy and myelopathy. Paraneoplastic neurological signs have been reported to precede the manifestation of cancer by months or years. The neurological symptoms may improve after the radical treatment of cancer. ${ }^{25} 6$

\begin{abstract}
LEL Employment Pension Fund, Palkkatilanportti 1, FIN-00240 Helsinki, Finland

J Juntunen

Tampere Regional Institute of

Occupational Health, PO Box 486, FIN-33101 Tampere, Finland

Abstract

Objective-To chart the subtle neurological abnormalities in patients with asbestosis relative to possible development of cancer.

Methods-In 1979-81 a standardised neurological examination was made of 115 patients with asbestosis who carried a high risk of occupational cancer and their cancer morbidity was analysed 15 years later.

Results-Slight disturbances of unknown aetiology were found in the central nervous system (CNS) of 33 and in the peripheral nervous system (PNS) of 41 patients. Of these 17 had disturbances of both the CNS and PNS. This cohort was followed up to the end of 1994. During this time 47 of the patients developed cancer. Statistical analyses showed that disturbances of the CNS such as psycho-organic syndrome, cerebellar dysfunction, and motor disturbances of unknown origin were significantly associated with cancer, whereas no such association was found for peripheral neuropathy. Interaction between the radiological progression of asbestosis and disturbances of the CNS was an even stronger predictor of cancer. Conclusions-It seems that slight disturbances of the CNS are predictors of development of cancer. Whether or not these disturbances are manifestations of involvement of a paraneoplastic nervous system or some factor associated with progression of asbestosis remains open.
\end{abstract}

Current knowledge on paraneoplastic phenomena is largely based on case reports and reports on patients with already diagnosed cancer. Long term prospective studies on this topic are lacking, and the value of paraneoplastic disturbance of the nervous system as a predictor or early sign of cancer is unclear. The present prospective clinical study was initially designed to elucidate this problem in a cohort of patients with asbestosis, a group with a high risk of cancer. ${ }^{7}$ The correlations of the neurological findings in these patients with some tumour markers, immunological variables, progression of asbestosis, and lung function were reported at the cross sectional stage of this study. ${ }^{8-10}$

\section{Subjects and methods}

Our study comprised a cohort of randomly selected patients with asbestosis consisting of typical Finnish patients with diagnosed pneumoconiosis and followed up regularly at the Department of Occupational Medicine in the Finnish Institute of Occupational Health, Helsinki, Finland. They were insulators, workers in asbestos cement product factories, workers in an asbestos quarry, and asbestos sprayers. As well as routine tests and examinations for pneumoconioses they were given a thorough standardised neurological examination by one of us (J) between November 1979 and March 1981.

The cohort comprised 105 men and 10 women. Their mean age at the time of the neurological examination was 56 (range 3276) years. They had been exposed to asbestos for an average of 21 (range 3-47) years. The mean time between the diagnosis of asbestosis and the neurological examination was eight (range 1-34) years. Seventy four of the 115 patients were also followed up radiographically. Two or three posteroanterior radiographs were taken in 1978-9, 1983-4, and 1986-7 and were independently classified according to the International Labour Office (ILO) 1980 classification $^{11}$ by three readers. Progression was estimated side by side. If the classification differed, a joint decision was made. For 30 $(40 \%)$ of the 74 patients the profusion of the small opacities had progressed during the follow up time (referred to as "progression" in this report).

NEUROLOGICAL EXAMINATION

The standardised neurological protocol applied in this study was developed at the Finnish Institute of Occupational Health primarily for epidemiological studies in occupational 
neurology. ${ }^{12}$ This neurological examination focused particularly on the subtle clinical signs of slight dysfunction of both the central nervous system(CNS) and peripheral nervous system (PNS). Difficulties in memory and recall, disturbances in drive and impulses, and general symptoms of organic brain damage were assessed. The examination of the PNS included tests for motor, sensory, and autonomic nerves. Muscular weakness and its distribution, muscle bulk, and muscle tone were examined. Muscle tendon reflexes were examined in a standardised manner. Symptoms of sensory disturbances were recorded by the sense of passive joint movement, by pinch press discrimination, by two point discrimination, and by the senses of direction, vibration, pain, touch, and temperature. Cranial nerves were tested in the usual way.

Autonomic symptoms such as postural vertigo, bladder dysfunction, gastrointestinal dysfunction, cardiac arrhythmias, sweating disturbances, and male impotence were asked about in detail. The examination of cerebellar dysfunction included tests which clearly indicated primary cerebellar damage (disturbances in coordination, muscle hypotonia, dysdiadochokinesis, cerebellar nystagmus, and cerebellar dysarthria). Disturbances in gait and station were examined by the routine Romberg's test, and also a modified version of it, and by finding the patients walking on heels, toes, and in tandem with the eyes either closed or open. The abnormal findings were graded according to their severity. All possible known aetiological factors for such findings were considered and registered. They included diabetes, heavy drinking, head trauma, cerebrovascular diseases, major psychiatric or neurological diseases, and medication.

Altogether 38 patients had disturbances of the CNS; 33 of these patients had disturbances of the CNS with an unknown aetiology (CNSNUD). Peripheral neuropathy was found in 44 patients, 41 having peripheral neuropathy with an unknown aetiology (PNS-NUD). Seventeen of these patients had both CNS-NUD and PNS-NUD. In the statistical analysis CNSNUD and PNS-NUD were considered separately. Altogether 58 patients had no signs of unknown disturbances of the nervous system (table 1).

REGISTERS AND STATISTICAL METHODS

The identification of people and the follow up of death and emigration for the years 1967-94 was done with the aid of the Population Register Centre with the unique identification number given to everyone residing in Finland since 1 January 1967 as the key.

Table 1 Neurological abnormalities of unknown aetiology at the beginning of the follow up of patients with asbestosis, and their cancer status at the end of follow up

\begin{tabular}{llccr}
\hline & \multicolumn{4}{c}{ Number (\%) of neurological abnormalities of unknown origin } \\
\cline { 2 - 5 } Cancer & Peripheral & Central & Both & None \\
\hline Lung cancer $(n=34)$ & $5(15)$ & $8(24)$ & $8(24)$ & $13(28)$ \\
Other cancer $(n=13)$ & $2(15)$ & $3(23)$ & $3(23)$ & $5(38)$ \\
No cancer $(n=68)$ & $17(25)$ & $5(7)$ & $6(9)$ & $40(59)$ \\
Total $(n=115)$ & $24(21)$ & $16(14)$ & $17(15)$ & $58(50)$ \\
\hline
\end{tabular}

The follow up for cancer was done automatically through the files of the Finnish Cancer Registry. It started on 1 January of the year after the year of the neurological examination. The follow up ended at the end of 1994 or when the first cancer (any other than nonmelanoma skin cancer) was diagnosed or when the patient died. The mean follow up time was 10 (range 0-15) years. The total number of person-years was 1112 .

Cox's model was used in the statistical analysis. The basic analysis was carried out with main effects only, but we also made a more detailed analysis to study possible interactions. The results were then interpreted with cross tabulations. The statistical analyses were carried out with BMDP statistical software (version 1993) on a VAX/VMS mainframe. The predictors used were age, sex, occupation, exposure time, smoking, ILO class at the beginning of the follow up, progression of small opacities during the follow up, and abnormalities of the CNS and PNS of unknown aetiology (PNS-NUD and CNSNUD).

\section{Results}

During the total (15 years) follow up time 47 of 115 patients $(41 \%)$ developed cancer, of which 34 had lung cancer. Other cancers were three mesotheliomas, two pancreatic and thyroid cancers and one oesophageal, colon, bile duct, renal, and brain cancer and one lymphosarcoma. Of the patients with asbestosis with cancer 29 had CNS-NUD or PNS-NUD (table 1).

The disturbances of the CNS were mainly slight psychoorganic syndromes, disturbances in gait and station, disturbances in cerebellar functions, and slight pyramidal and extrapyramidal disturbances. Many patients had several disturbances simultaneously. The disturbance in the PNS was predominantly slight sensorimotor polyneuropathy, mainly in the feet. Lung and other cancers did not differ from each other for neurological findings. The mean age of the patients with cancer was 55.4 (32-75 range) years, and that of the patients without cancer was 56.2 (34-76 range) years. There was no difference in the prevalences of PNS-NUD and CNS-NUD between the patients with cancer in the early stage of the follow up and those who developed it later. The figure shows the cumulative incidence of cancer during the follow up for the different groups of disturbances of the nervous system. The highest incidence was found for the patients with CNS-NUD, of whom $68 \%$ had cancer at the end of the follow up.

We fitted the Cox model in three different ways with the censoring limit set equal to 5,10 , or 15 years. The five year results are not given due to the few cancer patients (three cases).

\section{TEN YEAR FOLLOW UP}

For all cancer types the significant predictors of the model were radiographic progression of asbestosis $(P=0.0206)$ and CNS-NUD $(P=0.0036)$, which were positively correlated with the occurrence of cancer. Forty one per cent of the patients with progression and $10 \%$ 


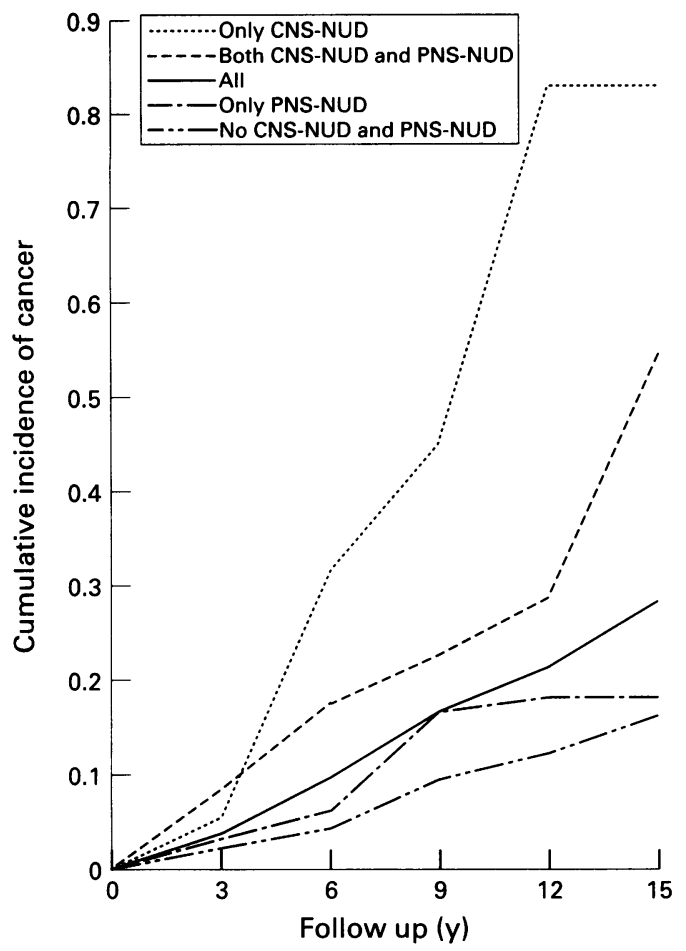

Age adjusted cumulative incidence of cancer for patients with asbestosis with CNS-NUD or PNS-NUD and those without such abnormalities.

of those without progression had cancer. Forty nine per cent of those with CNS-NUD and $25 \%$ of those without CNS-NUD developed cancer.

We then fitted the model for interaction between progression and CNS-NUD, which was then a significant predictor $(P=0.0002)$ When neither of these factors were present, $6 \%$ of the patients developed cancer; when only one was present $32 \%$ developed cancer; and, when both were present $67 \%$ developed cancer (table 2). In the interaction model occupation had a tendency to be a significant predictor $(P=0.0924)$. Thirty six per cent of the asbestos insulators and asbestos sprayers, $17 \%$ of the asbestos quarry workers, and $9 \%$ of the asbestos factory workers had cancer.

When only cases with lung cancer were studied, the results were analogous, the only

Table 210 Years of follow up of cancer patients: interaction pattern between progression of small opacities in the radiographs and central nervous system abnormalities with unknown aetiology (CNS-NUD)

\begin{tabular}{llll}
\hline \multirow{2}{*}{ Cancer } & \multicolumn{3}{l}{ Progression/CNS-NUD (n (\%)) } \\
\cline { 2 - 4 } & None & Only one & Both \\
\hline No cancer $(\mathrm{n}=56)$ & $32(94)$ & $21(68)$ & $3(33)$ \\
Cancer $(\mathrm{n}=18)$ & $2(6)$ & $10(32)$ & $6(67)$ \\
Total & $34(100)$ & $31(100)$ & $9(100)$ \\
\hline
\end{tabular}

Table 3 Fifteen years of follow up for cancers and lung cancer: interaction pattern between progression of small opacities in the radiographs and central nervous system abnormalities with unknown aetiology (CNS-NUD)

\begin{tabular}{llll}
\hline \multirow{2}{*}{ Cancer } & \multicolumn{3}{l}{ Progression/CNS-NUD (n (\%)) } \\
\cline { 2 - 4 } & None & Only one & Both \\
\hline No cancer & $30(88)$ & $17(56)$ & $2(22)$ \\
Cancer & $4(12)$ & $14(45)$ & $7(78)$ \\
Total & $34(100)$ & $31(100)$ & $9(100)$ \\
\hline
\end{tabular}

difference being that occupation was not included in the interaction model: CNS-NUD $(P=0.0809)$ and progression $(P=0.0036)$ were significant. Among the patients with CNSNUD, $40 \%$ had lung cancer, and for those without CNS-NUD the corresponding figure was $14 \%$. Thirty nine per cent of those with progression developed lung cancer, whereas $8 \%$ of the patients without progression developed it. The dependency pattern between interaction (CNS-NUD and progression, $P=0.0005$ ) and the follow up result was similar to the previous model.

FIFTEEN YEAR FOLLOW UP

When all the cancers were studied, the significant predictors were progression $(\mathrm{P}=0.0059)$, CNS-NUD $(\mathrm{P}=0.0994)$, and occupation $(P=0.0402)$. Fifty three per cent of the patients with progression and $21 \%$ of those without progression had cancer. Sixty three per cent of those with CNS-NUD and $24 \%$ of those without it had cancer. The cumulative incidence of cancer according to occupational group was $57 \%$ for asbestos sprayers, $36 \%$ for insulators, and $25 \%$ for factory workers. For the interaction model the result was the same, the significant predictors were an interaction of progression and CNS-NUD $(\mathrm{P}=0.0001)$ and occupation $(\mathrm{P}=0.0605)$

For lung cancer, both progression $(\mathrm{P}=0.0059)$ and CNS-NUD $(\mathrm{P}=0.0994)$ were again significant predictors, as was smoking $(\mathrm{P}=0.0402)$. If progression was present, $46 \%$ of the patients developed lung cancer, and, if not present, the proportion was $13 \%$. Also, if CNS-NUD was present, $53 \%$ developed lung cancer, as did $18 \%$ if it was not. Among the non-smokers no lung cancer was found, whereas $17 \%$ of the ex-smokers and $44 \%$ of the current smokers developed lung cancer. For the interaction model we obtained the same results - namely, that smoking $(P=0.0354)$ and an interaction of progression and CNS-NUD $(\mathrm{P}=0.0023)$ were significant.

The dependence patterns for both analyses were analogous to each other and also analogous to the results of the 10 year follow up (table 3).

\section{Discussion}

Analysis of the predictors associated with cancer morbidity in five year periods showed that CNS-NUD was significantly associated with development of cancer. This association was strengthened by the radiological progression of asbestosis and was not temporally related to the manifestation of cancer. There were no associations of age or disturbances of the PNS with cancer.

Neurological signs were found and scored systematically by an occupational neurologist with much experience with clinical epidemiology. The disturbances of the nervous system were generally slight, many of them falling into the category of normal variation in a routine clinical examination. Clinical experience with the proper interpretation of slight dysfunction of the CNS, such as psycho-organic alteration and cerebellar and balance disorders, cannot 
be overemphasised. ${ }^{13}$ As a method, comprehensive clinical examination provides a laborious but reliable way of detecting slight disturbances of the nervous system and is suitable for studies of small cohorts. ${ }^{12}$ The specificity and sensitivity of this method are probably close to optimal.

The prevalence of disturbances of the nervous system among our patients with asbestosis was higher than among several reference groups of patients examined at the same time (fibrosing alveolitis, suspected occupational solvent intoxication, and gynaecological carcinoma) and clearly higher than in a healthy population. ${ }^{7}$ Symptoms such as memory disturbances, tiredness, sensory disturbances, and clumsiness were more common among the patients with asbestosis and fibrosing alveolitis than among patients with chronic solvent poisoning. Therefore, it seems justified to conclude that the patients with asbestosis actually had an unusually high prevalence of disturbances of the nervous system. The possible explanations for this are that underlying asbestosis or some biological changes related to it somehow cause disturbances of the nervous system or there is a paraneoplastic phenomenon.

In this study radiological progression of asbestosis and CNS signs were both predictors of cancer. Together their strength was multiplicatory which indicates that a fibrosing process itself seems to have some role. Our earlier studies of this cohort did not show differences between the patients with and without involvement of the nervous system for several variables: pulmonary function, ${ }^{8}$ the tumour markers CEA, ferritin, $\beta$-2-microglobulin, antinuclear antibodies $\mathrm{C} 3$ and $C 4,{ }^{9}$ and circulating immune complexes. ${ }^{10}$ Thus neurological abnormalities are not related to these biological variables, many of which change in asbestosis.

Previous studies on disturbances of the nervous system in patients with cancer are confined entirely to cases with already diagnosed cancer. Therefore it is not possible to draw any conclusions about the possible predictive role of these disturbances for cancer. Case reports suggest that nervous system signs, particularly peripheral neuropathy, can precede the manifestation of cancer by months or years and that these signs can decrease after radical treatment of cancer. ${ }^{4-6}$ These findings suggest that cancer is somehow associated with disturbances of the nervous system, possibly through some immunological mechanism. ${ }^{14-16}$

Our statistical analyses showed that CNSNUD was a significant predictor of cancer
This unexpected finding is interesting and certainly deserves further study. The present study does not allow any conclusions to be drawn about the temporal association between disturbance of the nervous system and cancer or about the possible underlying mechanisms of this association. The complexity of the CNS makes it a susceptible target for many diffuse exogenous or endogenous agents, including toxic or immunological disorders. The paraneoplastic phenomenon is one possible mechanism, but it remains uncertain as an explanation.

This study has been financially supported by the Finnish Work Environment Fund and Medical Research Fund of the Tampere University Hospital. We thank all those colleagues at the Finnish Institute of Occupational Health who contributed to this study in its early stages.

1 Zuffa M, Kubanook J, Rusnak I, Mensatoris A, Horvath A Early paraneoplastic syndrome in medical oncology: clinicopathological analysis of 1694 patients treated over 20 years. Neoplasma 1984;31:231-6.

2 Elrington GM, Murray MN, Spiro SG, Newson-Davis J. Neurological paraneoplastic syndromes in patients with small cell lung cancer: a prospective survey of 150 patients. f Neurol Neurosurg Psychiatry 1991;54:764-7.

3 Clouston PD, DeAngelis LM, Posner JB. The spectrum of neurological diseases in patients with systemic cancer. Ann Neurol 1992;31:268-73.

4 Croft PB, Urich H, Wilkinson M. Peripheral neuropathy of sensorymotor type associated with malignant disease. Brain 1967;90:31-66.

5 Leinonen H, Sipilä R, Juntunen J. Paraneoplastic polyneuropathy associated with carcinoid tumour of cecum. Acta Med Scand 1982;212:183-4

6 Mitchell DM, Olczak A. Remission of a syndrome indistinguishable from motor neurone disease after resection of bronchial carcinoma. BMF 1979;2:176-7.

7 Juntunen J, Huuskonen M, Matikainen E, Kemppainen R, Suoranta $\mathrm{H}$, Tukiainen $\mathrm{P}$, et al. Asbestosis, the nervous system and cancer. Ann Acad Med 1984;13:353-60.

8 Korhonen $\mathrm{O}$, Juntunen J, Huuskonen M. Lung function and nervous system involvement in asbestosis. Eur $\mathcal{f} A p p l$ Physiol 1983;50:207-12.

9 Järvisalo J, Juntunen J, Huuskonen M, Kivistö H, Aitio A. Tumor markers and neurological signs in asbestosis. $A m \mathcal{F}$ Ind Med 1984;6:159-66.

10 Huuskonen $M$, Räsänen J, Juntunen J, Partanen $T$ Immunological aspects of asbestosis: patients neurological signs and asbestosis progression. Am f Ind Med 1984;5 461-9.

11 International Labour Office. Guidelines for the use of ILO international classification of radiographs of pneumoconioses rev ed. Geneva: ILO, 1980. (Occupational Safety and Health Series no 22 (Rev).)

12 Juntunen J. Neurological examination and assessment of the syndromes caused by exposure to neurotoxic agents. In: syndromes caused by exposure to neurotoxic agents. In: occupational health. Advances in the biosciences. London: Peroccupational health. Advances in

13 Juntunen J. Neurotoxic syndromes and occupational exposure to solvents. Environ Res 1993;60:98-111.

14 Anderson NE, Rosenblom MK, Graus F, Wiley RG, Posner JB. Autoantibodies in paraneoplastic syndromes associated with small-cell lung cancer. Neurology 1988;38:1391-8.

15 Dick DJ, Harris JB, Falkous G, Foster JB, Xuereb JH. Neuronal antinuclear antibody in paraneoplastic sensory neuronopathy. $\mathcal{F}$ Neurol Sci $1988 ; 85: 1-8$.

16 Dalmau J, Graus F, Rosenblom MK, Posner JB. Anti-Hu associated paraneoplastic encephalomyelitis/sensory neuropathy: a clinical study of 71 patients. Medicine (Baltimore) 1992;71:59-72. 